\title{
CD200/CD200R PAIRED POTENT INHIBITORY MOLECULES REGULATING IMMUNE AND INFLAMMATORY RESPONSES; PART II: CD200/CD200R POTENTIAL CLINICAL APPLICATIONS
}

\author{
Drahomíra Holmannová2, Martina Koláčková2, Kateřina Kondělková2, Pavel Kuneš ${ }^{1}$, Jan Krejsek², Ctirad Andrýs ${ }^{2}$
}

Charles University in Prague, Faculty of Medicine and University Hospital in Hradec Králové, Czech Republic: Department of Clinical Immunology and Allergology ${ }^{1}$, Department of Cardiac Surgery ${ }^{2}$

Summary: CD200 and its receptor were recognized as having the multiple immunoregulatory functions. Their immunoregulatory, suppressive, and tolerogenic potentials could be very effectively exploited in the treatment of many diseases, e.g. Alzheimer disease, rheumatoid arthritis, and allergy to name only some. Many research projects are aimed to develop clinically valuable methods being based on the structure and function of these paired molecules. In this review, we would like to introduce CD200/CD200R functions in a clinical context.

Key words: CD200; CD200R; Inflammation; Immunoregulation; Self-tolerance

\section{CD200/CD200R in osteology and osteoimmunology}

Bone metabolism is regulated in concert by osteoblasts and osteoclasts that direct bone production and resorption, respectively. Osteoblasts express CD200, whereas CD200R is preferentially expressed on osteoclasts. Osteoclasts are multinucleated cells formed by the fusion of macrophages on the bone surface. (30) Their over-activation results in osteoporosis associated with bone fractures. CD200/CD200R interaction increases the macrophage fusion and therefore potentiates bone resorption. It was determined that $\mathrm{CD} 200^{-/^{-}}$mice accumulate bone tissue more easily than $\mathrm{CD} 200^{+} /^{+}$mice, just by reducing the osteoclastoneogenesis. (47) Bone metabolism is also regulated by hormones, e.g. PTH, calcitriol, glucocorticoids, by prostaglandins, and cytokines. Proinflammatory cytokines such as IL- $1 \beta$, IL-6, and TNF $\alpha$ have been recognized as a potent negative regulator of osteoneogenesis. These cytokines promote osteoclast formation and increase their activity. CD200/CD200R engagement, on the contrary, diminishes the synthesis of proinflammatory cytokines and potentiates production of TGF- $\beta$, which supports the bone formation by enhancement of the recruitment and proliferation of osteoblast progenitors. The role of CD200/CD200R in osteogenesis so remains enigmatic as they stimulate both bone resorption (osteoclasts formation) and bone mass formation through the decrease of the expression of proinflammatory cytokines that participate in maintaining the delicate equilibrium between bone resorption and formation. (21)

\section{Triggering CD200/CD200R; A promissing approach to treat cancer}

The cell mediated reactivity such as NK, CD4 ${ }^{+}$Th1, $\mathrm{CD}^{+} \mathrm{CTL}$, macrophage subset M1, and TAMS (Tumor Infiltrating Macrophage), dendritic cells as well as cytokines - INF $\gamma$, chemotactic molecules, and CCR2 receptors binding MCP-1, MCP-2, MCP-3 chemokines, plays the main role in antitumor immunity. Opposite role supporting growth, invasivity, and cancer metastasis, is played by Th2, Treg, mast cells, macrophage subset M2, and MDSC (Myeloid Derivated Suppressor Cell); cytokines IL-10, TGF- $\beta$, VEGF, and inhibiting molecule CTLA-4 (Cytotoxic T-Lymphocyte Antigen-4). CD200/CD200R interaction is an orchestrator of iTreg, Teff, and macrophage subset Gr1 polarization, whereas limits the number, cytolytic activity, and unresponsiveness to INF $\gamma$ stimulation of NK and of TIL cells (Tumor Infiltrating Lymphocyte). (5) CD200/CD200 ligation creates the immune-suppressive tumor microenvironment. Higher expression of CD200 on tumor cells is described in both solid and hematopoetic malignancies. (29) CD200 expression is demonstrated in $78 \%$ cases of CLL and can be used to confirm the diagnosis of primary cutaneous B-cell lymphomas. It may also help to distinguish for instance between CLL vs. B cell lymphoma. $(6,31)$ The presence of CD200 is accented on less differentiated immature cells, mainly on cancer stem cells, termed as initiators of tumorigenesis, trigger of relapses and metastasis after antitumor therapy. (16) It was uncovered by in vitro and in vivo studies of melanoma, squamous carcinoma, and breast cancers that cancer aggressivity, proliferation and metastasis potential is positively correlated with CD200R expression and with the level of soluble form 
of CD200, which enhances the inhibition of leukocytes in the tumor microenvironment. $(13,41)$ It was discovered that TILs lose their reactivity because of CD200 overexpression on the tumor cells. Application of anti-CD200 monoclonal antibodies restores cytotoxic potential of TILs. (8) It is acknowledged that breaking the suppression established by CD200/CD200R interaction intensifies success and avoid failure of antitumor therapy. Treatment by PMA (Phorbol 12-Myristate 13-Acetate) down-regulates CD200 expression and thus improves defense mediated by lymphokine-activated killer cells during antitumor therapy. (48) Interest of researchers is focused on the synthesis of anti-CD200s and their application for treatment of cancer. (19) It was established that application of anti-CD200 monoclonal antibodies restores a cytotoxic potential of TILs that results in tumor cell destruction. (8) The most effective in breaking down the inhibition established by CD200/ CD200R activation is the anti-G2G4 molecule $\left(\mathrm{C}_{\mathrm{H}} 1\right.$ constant domain of IgG2 joined with constant domains $\mathrm{C}_{\mathrm{H}} 2$, $\mathrm{C}_{\mathrm{H}} 3$ of IgG4) that binds CD200R and eliminates the leukocyte suppression. (18)

\section{vOX2 viral homologue of CD200 and viral escape}

Antiviral immunity comprises both humoral (INF $\alpha, \beta$, $\gamma, \omega$, complement, specific antibodies) and cell mediated immunity (CTL, NK). Viruses that can persist in a host such as HSV, CMV, myxoma virus, measles virus, encode viral CD200 homologue, which is expressed on the surface of infected cells. It could be assumed, that vOX2 participate in the immune system inhibition. Opposite situation occurs at the onset of infection. It was determined in vitro that vOX2 of Kaposi's sarcoma-associated herpesvirus activates the myeloid cells and increases the synthesis of inflammatory cytokines. This strategy of viral escape supports cytokine-mediated neoangiogenesis, which is characteristic for Kaposi's sarcoma. Moreover, the recruitment of leukocytes to the initial site of infection enhances virus dissemination throughout the infected host. (4) However, during infection vOX2 mediates immune inhibition that is accompanied with down-regulated phagocytosis and antigen presentation. (36) vOX2 expression is typical for myxoma virus (M141R). Its vOX2 shows 40\% (HSV-8 $20 \%$ ) homology to human CD200. M141R strain does not alter macrophage activation and migration. Inhibition is targeted to the iNOS synthesis and T cells activation accompanied with INF $\gamma$ decrease. (1) Rhesus rhadinovirus R15 vOX2 molecule inhibits translation of the TNF- $\alpha$ mRNA in monocytes activated by INF $\gamma$. (20) Homologue of CD200 protects viruses against the immune system. On the other site, CD200 regulates the immune response intensity during viral infection and protects tissues against unwanted damage. In vivo experiments with CD200- ${ }^{-}$mice revealed that inoculation with influenza virus resulted in massive inflammatory response and serious damage of the lung parenchyma because of infiltration by leukocytes and their production of iNOS, IL-6, TNF $\alpha$, MIP-1 $\alpha$. It has been proven that administration of CD200 agonists diminished peribronchial leukocyte infiltration. $(15,35)$ Administration of CD200Fc during uveitis caused by HSV also weakens the infiltration of the tissue by leukocytes, reduces the level of proinflammatory cytokines, and potentiates differentiation of T cells to Treg FoxP3 ${ }^{+}$cells in sentinel lymph nodes. Thus in this regard, the risk of corneal neovascularization is diminished. (37)

\section{The role of the regulatory $\mathrm{CD200/CD200R}$ in nervous system and autoimmune pathology}

The CNS comprises many cell types such as neurons, glial cells (microglias constituting 20\% of the total glial population), and astrocytes. Microglia are termed as the resident macrophages of the CNS. These cells, in contrast to astrocytes, oligodendrocytes, which originate from ectoderm, are derived from mesoderm. Myeloid progenitors, which arise from the yolk sack, invade the CNS during embryogenesis and give rise to microglia population. Microglia are known to share many features with other myeloid cells, especially with macrophages. Upon activation, macrophages and microglia exert very similar functions, cytokine release and de novo cytokine synthesis of TNF $\alpha$, IL-1 $\beta$, IL-6, IL-1, MCP-1, MIP-1 $\alpha$, fractalkin, iNOS, but microglia differ from macrophages in several ways, e.g. produce lower levels of superoxide dismutase. $(25,32)$ Findings regarding microglia functions show that microglias play a crucial role in the pathogenesis of neurodegenerative, chronic inflammatory diseases of the CNS, but microglia also express GLT-1 (Glutamate Transporter) which protects the CNS from glutamate toxicity, participates in microbial immunity (e.g. Toxoplasma gondii), supports synaptic functions, removes neuronal debris, and amyloid $\beta$, which accumulation is linked to Alzheimer disease. (2) Microglia express CD68 (marker of macrophage lysosomal activity), TLR, and are able to present antigen. (25) There is the evidence that CD200/CD200R engagement promotes microglia quiescence. Expression of CD200R on microglia, and CD200 on neurons is increased after the CNS alteration caused either by trauma, surgery or ischemia. (27) Neurodegenerative diseases are, on the contrary, characterized by the decreased expression of CD200. (45) It is confirmed that down regulated expression of CD200 (not CD200R) is negatively correlated with longterm microglia activation, e.g. in Parkinson and Alzheimer diseases. CD200/CD200R engagement has the capacity to decelerate the progression of the previously mentioned neurodegenerative disorders. (46) It was discovered that CD200/CD200R expression on MDMs (MonocyteDerived Macrophages) in patients suffering from Parkinson disease is not increased after cultivation with injured or dead neurons. Under physiological circumstances, these stimuli strengthen the expression of CD200 and CD200R. 
(24) Patients suffering from Alzheimer disease also show significantly lower expression of CD200, mainly in hippocampus, superior frontal gyrus, and inferior temporal gyrus. $(22,44)$ It was determined that CD200m RNA expression is enhanced by IL-4 and IL- 13, but production of IL-4 decreases with age so does the IL-4 dependent expression of CD200m RNA. (23) Retina as an integral part of the neural system utilizes the same principles to control inflammation by CD200/CD200R interaction. It was found that isoform CD200RLa potentiates IL-10 production. (42) Recent research described the involvement of CD200R in microglia activation in experimental ocular hypertension. Obtained data showed that CD200/CD200R activation may reduce retinal cell loss in glaucoma. (43) Multiple sclerosis also belong among serious neurological diseases, which is in majority of patients clinically characterized by a relapsing-remitting course followed by a progressive phase in which neural degenerative changes, demyelinization with axonal and neuronal injury are typically found. Multiple sclerosis is recognized as an autoimmune disease. CD200/CD200R interaction is an important mechanism limiting autoimmune inflammation, as has been proved by the number of studies, using animal models of multiple sclerosis, rheumatoid arthritis, and autoimmune uveitis, respectively. In these experiments CD200/CD200R interaction promotes immune tolerance or nonresponsiveness to autoantigens. Experimental Autoimmune Encephalomyelitis (EAE) is an animal model of multiple sclerosis induced in genetically prone mice by the administration originally crude extracts from the brain tissues, nowaday by molecularly fully characterized components of these extracts such as MOG (Myelin Oligodendrocyte Glycoprotein). The development of immunopathology is only possible, if autoantigens are administered in the complete Freund adjuvans. During EAE, the inflammatory response is suppressed by the interaction of CD200/CD200R, which inhibits the microglia activation. CD200R agonist CD200Fc (solubilized forms of the CD200 molecules) administered to mice during the chronic phase of EAE are able to reduce the severity of EAE and slow the disease progression. CD200/CD200R interaction inhibits microglia/ macrophage accumulation in the CNS by reducing surface adhesion molecules expression such as VLA-4 (Very Late Antigen-4) and LFA-1 (Lymphocyte Function-Associated antigen-1) integrins. Moreover, CD200R activation is followed by decreased expression of MHC II, CD80, and reduced proinflammatory cytokines synthesis. (22) Studies of autoimmune diseases in context of CD200/CD200R interaction also involve rheumatoid arthritis. Etiology and dynamics of rheumatoid arthritis is studied in vivo using the model of collagen induced arthritis in mice (DBA/1). (12) Immunization by collagen II evokes arthritis, which severity is dependent on the CD200 mediated repression. It has been proven that $\mathrm{CD} 200^{-} /$mice showed rapid progression of RA. (40) Progression of RA can be slowed or even stopped after CD200Fc administration. CD200Fc also affects cytokine synthesis, and is successful in lowering the level of proinflammatory cytokines (TNF $\alpha, \mathrm{IL}-1 \beta$, MMP-13, and INF $\gamma$ ). If the administration of CD200Fc preceded collagen immunization, or followed it immediately after application, the mice were protected against arthritis development. (39)

\section{CD200 and allograft protection}

The immune system has an ability to recognize genetically different $\mathrm{MHC}$ molecules.

MHC proteins are highly polymorphic. Disparity of donor and patient MHCs contributes to the graft rejection. The immune response includes activation of APC, T cells Th1, Th2 regulatory subset, CTL, NK, up-regulation of MHC I/II expression, synthesis, and releasing of proinflammatory cytokines, and chemokines. Many studies deal with CD200/CD200R interaction and show its influence on the graft acceptance. Transgenic mice that overexpress CD200 are used for experiments to appreciate its role in rejection mechanisms. (49) CD200R stimulated DCs positively modulate alloimmune tolerance; promote polarization to the Th2 cytokine production, promote differentiation of $\mathrm{T}$ cell to iTreg, reduce CTL and NK activity, potentiate IL-10, TGF- $\beta$, which supports differentiation of naive $\mathrm{T}$ cell to $\mathrm{CD} 4^{+} \mathrm{CD} 25^{+} \mathrm{Fox}^{+}$Treg, production and increase a chance of graft acceptance. It is confirmed that the application of anti-CD200 monoclonal antibodies results in graft rejection. (9) Recently, the hybrid molecule CD200Fc(Gly)6TGF $\beta$ was prepared by Gorczynski et al. (10) It can suppress activity of leukocytes at 20-100-fold lower concentrations than CD200 or TGF- $\beta$ alone, or in their combination. It is concluded that this strong inhibitory effect is mediated by at least two types of interactions. CD200Fc(Gly)6TGF $\beta$ has the capacity to bind T cell through TGF- $\beta$ and simultaneously APC through CD200R1. (11)

\section{CD200 prevents spontaneous fetal loss}

CD200/CD200R interaction has been proved to possess a protective properties against spontaneous fetal loss, which is primarily driven by Th1 T cells producing INF $\gamma$, and TNF $\alpha$. Stimulated Th1 T cells increase fibrinogen-like protein 2 and thrombin expression, thus generate prothrombogenic and proinflammatory microenvironment. CD200 molecules are highly expressed on trophoblasts and inhibit the cell-mediated immune response targeted against the fetus and induce immune tolerance in this way. $(4,50)$

\section{CD200/CD200R and the skin}

The skin is in a permanent contact with the environment and thus is exposed to a large number of external pathogens. The regulation of immune reactions in the skin is tuned to maintain the balance between defense and tolerance. CD200/CD200R interaction attenuates 
inflammatory responses and promotes tolerance. It was for instance reported that in CD200 ${ }^{-/}$mice UVB-irradiation induced tolerance to haptens could not be established. CD200 expression in the skin is described on Langerhans cells, mast cells, macrophages, $\gamma \delta^{+} \mathrm{T}$ cells, and on $\mathrm{CD}^{-}$ MHC $\mathrm{II}^{-}$epidermal cells (EC) that are mainly localized with $\mathrm{K} 14^{+}$keratinocytes in the outer root sheath of the hair follicle. (26) It was observed that in absence of CD200 intra- and perifollicular inflammation is more pronounced even after a mild insult. Cells expressing keratin 15, CD200, CD34, CD271 show high self-renewal and proliferative capacity and have a potential to prevent alopecia. CD200 keratinocytes and Langerhans cells are able to protect the hair follicle and mitigate damage caused by inflammation. CD200 is supposed to be the provider of immune privilege for bulge stem cells. $(7,33)$

\section{CD200/CD200R and cardiovascular system}

Immunohistochemical methods and flow cytometry were used to localize CD200 expression on endothelial cells. The highest expression was found in small bloodvessels and capillaries, in the area where endothelial cells and leukocytes are in the intimate contact and leukocytes transmigrate to injured tissue. On the contrary, the weak expression was shown in arteries and aorta. (17) Cardiac surgery is associated with ischemia that contributes to the systemic inflammatory response. Ischemia and inflammatory markers in cardiac surgery are CRP, PTX3 (pentraxin), troponin, myoglobin, CK-MB, myeloperoxidase, FABP (heart-type Fatty Acid-Binding Protein), PAPP-A (Pregnancy-Associated Plasma Protein A), and IL-8 to name only some. Leukocyte depletion during surgery reduces inflammation as well as CD200/CD200R interaction. Expression of CD200R on macrophages was increased after the surgery, meanwhile postoperative expression of CD200R on blood monocytes was decreased. $(18,34) \mathrm{CD} 200$ in cardiovascular system attenuates inflammatory reactions, protects tissue against damage and potentiates the production of TGF- $\beta$, that support heart tissue reparation and start regeneration.

\section{Quiscence of mast cells and basophils by CD200/CD200R interaction}

The crucial role in allergic inflammation is given to the mast cells. They are distributed in body tissues and serve as sentinel cells producing the plethora of proinflammatory mediators after being activated by invading harm stimuli. They are strategically accumulated in perivascular areas, beneath epithelial layers of the skin and mucosa of airways and gut and drive $\mathrm{T}$ cell polarisation to Th2, followed by $\mathrm{B}$ cells activation and immunoglobulin production. Mast cells express high affinity FceRI receptors, which are occupied by IgE immunoglobulines. (28) The cross-linking of FceRI by $\operatorname{IgE}$ and antigen initiates activation cascades that result in mast cells degranulation and release of preformed ,substances“ such as histamine, heparin, various proteases, and de novo synthesis of cytokines, leukotrienes, prostaglandins, and growth factors. Both mast cell FceRI-induced degranulation and synthesis and secretion of cytokines (TNF $\alpha$, IL-13) is repressed by CD200/CD200R interaction. (3) Zhang et al. developed in vitro model of mast cell degranulation to study inhibitory mechanisms of CD200/CD200R interaction. They confirmed that CD200R reduces degranulation and IL-13 and TNF $\alpha$ secretion. If the cells were treated with CD200R agonists, a suppression was achieved. CD200/CD200R activation thus regulates extent and duration of allergic response. (51) Basophils are another type of leukocytes involved in allergic reactions. Like mast cells, they express high affinity FceRI receptors. Aggregation of FceRI bound to IgE by polyvalent antigen is followed by basophil activation, degranulation, mediator release, and increased migration into tissue. Recently, it has been described that density of CD200R expression on basophils depends on serum IgE and IL-4 serum level and rapidly increases after basophils activation. CD200R can serve as a marker of basophil activation. CD200/ CD200R engagement on basophils attenuates the allergic inflammation similarly to mast cells.

\section{Conclusion}

CD200/CD200R interaction renders the immune system unresponsive, establishes peripheral immunological tolerance. All studies to date have confirmed that the CD200/CD200R interaction regulates myeloid cell activity, attenuates inflammatory reactions both, cell mediated and humoral, in a vulnerable damaged tissue. CD200/CD200R engagement reveals a great clinical significance. Inhibition induced by CD200/CD200R may be beneficial in the treatment of autoimmune, inflammatory, and hypersensitivity diseases where the excessive response of the immune system may be harm for the tissues. Previous studies in vitro or in vivo using mouse models demonstrated that CD200/CD200R positively influences progression of diseases. CD200/CD200R interaction seems to be very promising in transplantation medicine to establish immunotolerance and to protect the graft from rejection. On the contrary, in tumors growth or viral infections, a blockade of CD200R limits suppression induced by tumor cells or $\mathrm{vOX}$ with subsequent activation of the immune responses that allows to overcome disease. Only a few research projects deal with a role of CD200/CD200R in an association with cardiovascular, gastrointestinal, or urogenital systems, respectively. It is therefore questionable whether there would not be possible to use their inhibitory ability in the treatment of e.g. nonspecific intestinal inflammation. Immunobiological activities of CD200/CD200R interactions are shown in Fig. 1 and described in legend. 


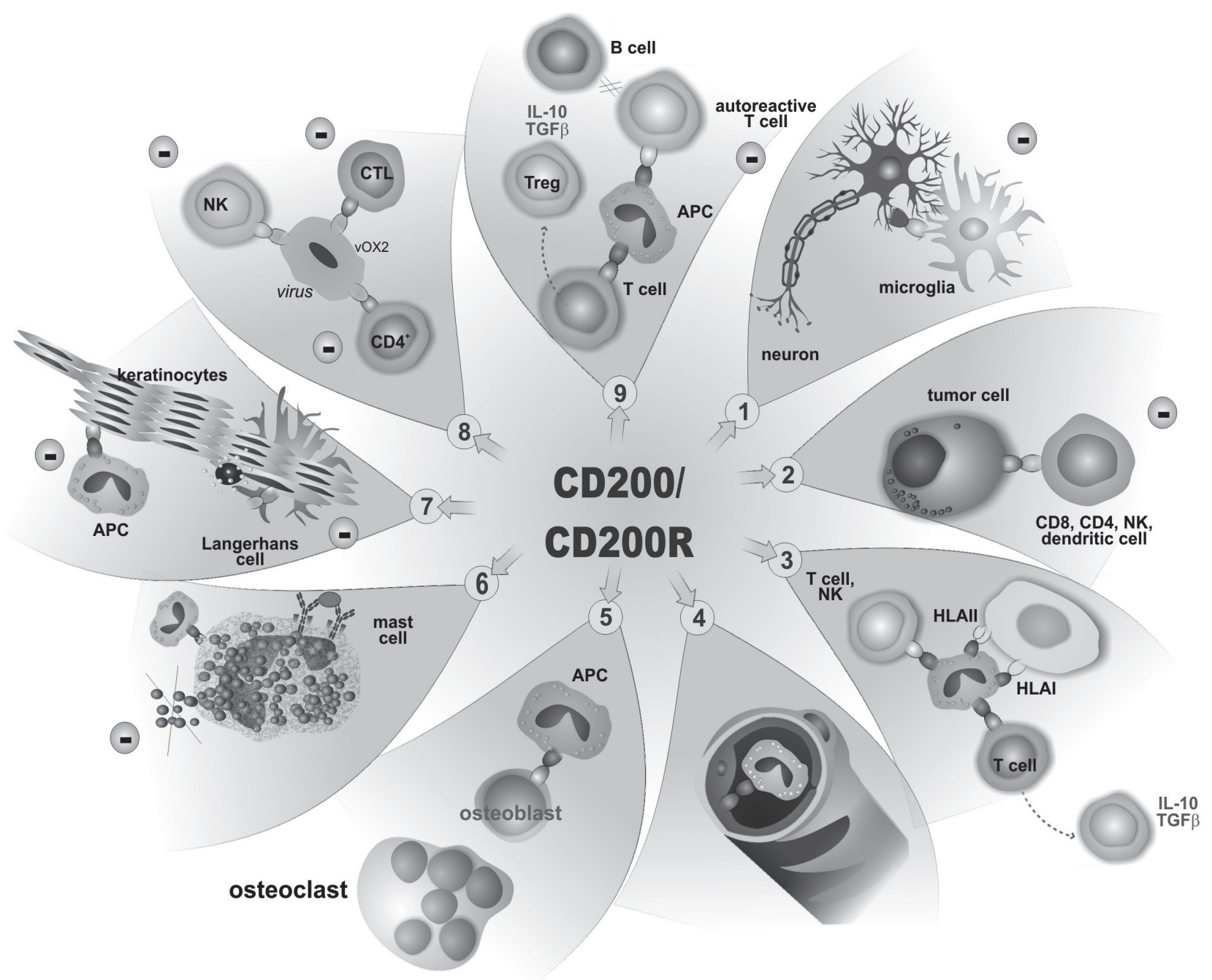

Fig. 1: CD200/CD200R interaction in clinical context

\section{Legend}

Inhibition driven by CD200/CD200R engagement affects immune reactivity in many tissues.

1) Brain and nerves - microglias inhibition diminishes the inflammation, decelerates progression of neurodegenerative diseases, protects neurons against damage caused by trauma, operation or stroke.

2) Oncology - high expression of CD200 on tumor cells promotes an immune suppressive tumor microenvironment and thus induces leukocytes unresponsiveness.

3) Transplantology - CD200/CD200R interaction inhibits the immune system response and potentiates graft acceptance.

4) Cardiovascular system - expression of CD200/ CD200R is documented on the endothelium, the smaller diameter an artery has, the higher expression of CD200 the artery shows. Intensity of CD200/
CD200R expression on leukocytes is influenced by cardiovascular surgery.

5) Osteology - CD200/CD200R interaction acting both on bone resorption and bone formation.

6) Allergy - CD200/CD200R attaching stabilized mast cells and basophils and prevent from degranulation in response to IgE cross-linking of FceRI receptors.

7) Dermatology - CD200/CD200R engagement suppresses resident tissue and to skin migrating leukocytes, prevents the development of eczema, dermatitis...

8) Viral infection - Viruses encode the CD200 homologue which is expressed on infected cells. It restrains leukocytes response to viral infection and enhances the viral persistence in the infected cells.

9) Autoimmunity - CD200/CD200R inhibits the immune system autoreactivity, supports the self tolerance, differentiation of $\mathrm{T}$ cells to Treg subset, and IL-10, TGF- $\beta$ synthesis. 


\section{Acknowledgements}

This work was supported by Charles University in Prague, Faculty of Medicine in Hradec Králové, project "PRVOUK" P37/10.

\section{Abbreviations}

\section{APC - Antigen-presenting cell}

CCR2 - Receptor for CC chemokines

CLL - Chronic lymphocytic leukemia

$\mathrm{CRP}$ - C-reactive protein

CTL - Cytotoxic T lymphocytes

CTLA-4 - Cytotoxic T-lymphocyte antigen-4

EAE - Experimental autoimmune encephalomyelitis

HSV - Herpes simplex virus

iNOS - Inducible nitric oxide synthase

LFA-1 - Lymphocyte function-associated antigen-1

MCP-1 - Monocyte chemoattractant protein-1

MIP-1 - Macrophage inflammatory protein-1

MMPs - Matrix metalloproteinases

NK - Natural killer

PTH - Parathormon

PTX3 - Pentraxin-related protein

Teff - T effector cells

TIL - Tumor infiltrating lymphocytes

VEGF - Vascular endothelial growth factor

VLA-4 - Very late antigen-4

vOX2 - Viral CD200 homologue

\section{References}

1. Cameron CM, Barrett JW, Liu L, Lucas AR, McFadden G. Myxoma virus M141R expresses a viral CD200 (vOX-2) that is responsible for down-regulation of macrophage and T-cell activation in vivo. J Virol 2005; 79(10): 6052-67.

2. Chao CC, Gekker G, Hu S, Peterson PK. Human microglial cell defense against Toxoplasma gondii. The role of cytokines. J Immunol. 1994 Feb; 152(3) 1246-52.

3. Cherwinski HM, Murphy CA, Joyce BL, et al. The CD200 receptor is a novel and potent regulator of murine and human mast cell function. J Immunol 2005; 174(3): 1348-56.

4. Chung YH, Means RE, Choi JK, Lee BS, Jung JU. Kaposi’s sarcoma-associated herpesvirus OX2 glycoprotein activates myeloid-lineage cells to induce inflammatory cytokine production. J Virol 2002; 76(10): 4688-98.

5. Coles SJ, Wang EC, Man S, et al. CD200 expression suppresses natural killer cell function and directly inhibits patient anti-tumor response in acute myeloid leukemia. Leukemia 2011; 25(5): 792-9.

6. Dorfman DM, Shahsafaei A. CD200 (OX-2) membrane glycoprotein expression in B cell-derived neoplasms. Am J Clin Pathol 2010; 134(5): 726-33.

7. Garza LA, Yang CC, Zhao T, et al. Bald scalp in men with androgenetic alopecia retains hair follicle stem cells but lacks CD200-rich and CD34-positive hair follicle progenitor cells. J Clin Invest 2011; 121(2): 613-22.

8. Gorczynski RM, Chen Z, Diao J, et al. Breast cancer cell CD200 expression regulates immune response to EMT6 tumor cells in mice. Breast Cancer Res Treat 2010; 123(2): 405-15.

9. Gorczynski RM, Chen Z, He W, Khatri I, Sun Y, Yu K, Boudakov I. Expression of a CD200 transgene is necessary for induction but not maintenance of tolerance to cardiac and skin allografts. J Immunol 2009; 183(3): 1560-8.

10. Gorczynski R, Chen Z, Shivagnahnam S, Taseva A, Wong K, Yu K, Khatri I. $\mathrm{CD} 200 \mathrm{Fc}(\mathrm{Gly}) 6 \mathrm{TGF} \beta$ suppresses transplant rejection and MLCs in vitro. J Immunol 2010; 184, 49.15.

11. Gorczynski R, Chen Z, Shivagnahnam S, Taseva A, Wong K, Yu K, Khatri I. Potent immunosuppression by a bivalent molecule binding to CD200R and TGFbeta. Transplantation 2010; 90(2): 150-9.

12. Gorczynski RM, Chen Z, Yu K, Hu J. CD200 immunoadhesin suppresses collagen-induced arthritis in mice. Clin Immunol 2001; 101(3): 328-34.
13. Gorczynski RM, Clark DA, Erin N, Khatri I. Breast cancer cells CD200 expression regulates immune response to EMT6 tumor cells in mice. Breast Cancer Res Treat 2010; 123(2): 405-15.

14. Gorczynski RM, Hadidi S, Yu G, Clark DA. The same immunoregulatory molecules contribute to successful pregnancy and transplantation. Am J Reprod Immunol 2002; 48(1): 18-26.

15. Jiang-Shieh YF, Chien HF, Chang CY, et al. Distribution and expression of CD200 in the rat respiratory system under normal and endotoxin-induced pathological conditions. J Anat 2010; 216(3): 407-16.

16. Kawasaki BT, Mistree T, Hurt EM, Kalathur M, Farrar WL. Co-expression of the tolerogenic glycoprotein CD200, with markers for cancer stem cells. Biochem Biophys Res Commun 2007; 364(4): 778-782.

17. Ko YC, Chien HF, Jiang-Shieh YF, et al. Endothelial CD200 is heterogeneously distributed, regulated and involved in immune cell-endothelium interactions. J Anat 2009; 214(1): 183-95.

18. Krejsek J, Kolackova M, Mandak J, et al. Expression of CD200/CD200R regulatory molecules on granulocytes and monocytes is modulated by cardiac surgical operation. Perfusion 2010; 25(6): 389-97.

19. Kretz-Rommel A, Qin F, Dakappagari N, Cofiell R, Faas SJ, Bowdish KS. Blockade of CD200 in the presence or absence of antibody effector function: implications for anti-CD200 therapy. J Immunol 2008; 180(2): 699-705.

20. Langlais CL, Jones JM, Estep RD, Wong SW. Rhesus rhadinovirus R15 encodes a functional homologue of human CD200. J Virol 2006; 80(6): 3098-103.

21. Lee L, Liu J, Manuel J, Gorczynski RM. A role for the immunomodulatory molecules CD200 and CD200R in regulating bone formation. Immunol Lett 2006; 105(2): $150-8$.

22. Liu Y, Bando Y, Vargas-Lowy D, et al. CD200R1 agonist attenuates mechanisms of chronic disease in a murine model of multiple sclerosis. J Neurosci 2010 30(6): 2025-38.

23. Lue LF, Kuo YM, Beach T, Walker DG. Microglia activation and anti-inflammatory regulation in Alzheimer's disease. Mol Neurobiol 2010; 41(2-3): $115-28$

24. Luo XG, Zhang JJ, Zhang CD, Liu R, Zheng L, et al. Altered regulation of CD200 receptor in monocyte-derived macrophages from individuals with Parkinson's disease. Neurochem Res 2010; 35(4): 540-7.

25. Lynch MA.The multifaceted profile of activated microglia. Mol Neurobiol 2009; 40(2): 139-56

26. Matsue H. CD 200-mediated regulation of skin immunity. J Invest Dermatol $2005 ; 125(6): 2691-8$

27. Matsumoto H, Kumon Y, Watanabe H, et al. Expression of CD200 by macrophage-like cells in ischemic core of rat brain after transient middle cerebral artery occlusion. Neurosci Lett 2007; 418(1): 44-8.

28. Miyajima I, Dombrowicz D, Martin TR, Ravetch JV, Kinet JP, Galli SJ. Systemic anaphylaxis in the mouse can be mediated largely through IgG1 and Fc gammaRIII. Assessment of the cardiopulmonary changes, mast cell degranulation, and death associated with active or IgE- or IgG1-dependent passive anaphylaxis. J Clin Invest 1997; 99(5): 901-14.

29. Moreaux J, Veyrune JL, Reme T, De Vos J, Klein B. CD200: a putative therapeutic target in cancer. Biochem Biophys Res Commun 2008; 366(1): 117-22.

30. Oursler MJ. Recent advances in understanding the mechanisms of osteoclast precursor fusion. J Cell Biochem 2010; 110(5): 1058-62.

31. Pallasch CP, Ulbrich S, Brinker R, Hallek M, Uger RA, Wendtner CM. Disruption of T cell suppression in chronic lymphocytic leukemia by CD200 blockade. Leuk Res 2009; 33(3): 460-4.

32. Prinz M, Mildner A. Microglia in the CNS: immigrants from another world. Glia. 2011; 59(2): 177-87.

33. Rosenblum MD, Yancey KB, Olasz EB, Truitt RL. CD200, a "no danger" signal for hair follicles. J Dermatol Sci 2006; 41(3): 165-74

34. Rubino AS, Serraino GF, Mariscalco G, Marsico R, Sala A, Renzulli A. Leukocyte depletion during extracorporeal circulation allows better organ protection but does not change hospital outcomes. Ann Thorac Surg 2011; 91: 534-540.

35. Rygiel TP, Rijkers ES, de Ruiter T, et al. Lack of CD200 enhances pathological $\mathrm{T}$ cell responses during influenza infection. J Immunol 2009; 183(3): 1990-6.

36. Salata C, Curtarello M, Calistri A, et al. vOX2 glycoprotein of human herpesvirus 8 modulates human primary macrophages activity. J Cell Physiol 2009; 219(3): 698-706.

37. Sarangi PP, Woo SR, Rouse BT. Control of viral immunoinflammatory lesions by manipulating CD200:CD200 receptor interaction. Clin Immunol 2009; 131(1): $31-40$.

38. Shiratori I, Yamaguchi M, Suzukawa M, et al. Down-regulation of basophi function by human CD200 and human herpesvirus-8 CD200. J Immunol 2005; 175(7): 4441-9.

39. Simelyte E, Alzabin S, Boudakov I, Williams R. CD200R1 regulates the severity of arthritis but has minimal impact on the adaptive immune response. Clin Exp Immunol 2010; 162(1): 163-8

40. Simelyte E, Criado G, Essex D, Uger RA, Feldmann M, Williams RO. CD200$\mathrm{Fc}$, a novel antiarthritic biologic agent that targets proinflammatory cytokine expression in the joints of mice with collagen-induced arthritis. Arthritis Rheum 2008; 58(4): 1038-43. 
41. Stumpfova M, Ratner D, Desciak EB, Eliezri YD, Owens DM. The immunosuppressive surface ligand CD200 augments the metastatic capacity of squamous cell carcinoma. Cancer Res 2010; 70(7): 2962-72.

42. Taylor N, McConachie K, Calder C, Dawson R, Dick A, Sedgwick JD. Enhanced tolerance to autoimmune uveitis in CD200-deficient mice correlates with a pronounced Th2 switch in response to antigen challenge. J Immunol 2005; 174(1) $143-54$.

43. Taylor S, Calder CJ, Albon J, Erichsen JT, Boulton ME, Morgan JE. Involvement of the CD200 receptor complex in microglia activation in experimental glaucoma. Exp Eye Res 2011; 92(5): 338-43.

44. Torrero MN, Larson D, Hübner MP, Mitre E. CD200R surface expression as a marker of murine basophil activation. Clin Exp Allergy. 2009; 39(3): 361-9.

45. Walker DG, Dalsing-Hernandez JE, Campbell NA, Lue LF. Decreased expression of CD200 and CD200 receptor in Alzheimer's disease: a potential mechanism leading to chronic inflammation. Exp Neurol 2009; 215(1): 5-19.

46. Wang XJ, Ye M, Zhang YH, Chen SD. CD200/CD200R regulation of microglia activation in the pathogenesis of Parkinson's disease. J Neuroimmune Pharmacol 2007; 2(3): 259-64.
47. Weiguo Cui, Esteban Cuartas, Juan Ke, et al. CD200 and its receptor, CD200R, modulate bone mass via the differentiation of osteoclasts. Proc Natl Acad Sci USA 2007 ; 104(36): 14436-41.

48. Wong KK, Khatri I, Shaha S, Spaner DE, Gorczynski RM. The role of CD200 in immunity to B cell lymphoma. J Leukoc Biol 2010; 88(2): 361-72.

49. Yu K, Chen Z, Wang S, Gorczynski R. Decreased alloreactivity using donor cells from mice expressing a CD200 transgene under control of a tetracycline-inducible promoter. Transplantation 2005; 80(3): 394-401.

50. Yu G, Sun Y, Foerster K, Manuel J, et al. LPS-induced murine abortions require $\mathrm{C} 5$ but not $\mathrm{C} 3$, and are prevented by upregulating expression of the CD200 tolerance signaling molecule. Am J Reprod Immunol 2008; 60(2): $135-40$

51. Zhang S, Cherwinski H, Sedgwick JD, Phillips JH. Molecular mechanisms of CD200 inhibition of mast cell activation. J Immunol 2004; 173(11): 6786-93.

Received: $16 / 08 / 2011$

Accepted in revised form: 05/03/2012

\section{Corresponding author:}

Jan Krejsek, Charles University in Prague, Faculty of Medicine and University Hospital Hradec Králové, Department of Clinical Immunology and Allergology, Czech Republic; e-mail: krejsek@fnhk.cz 